\title{
Multi-modal imaging of long-term recovery post-stroke by positron emission tomography and matrix-assisted laser desorption/ionisation mass spectrometry
}

DOI:

$10.1002 / \mathrm{rcm} .8090$

\section{Document Version}

Accepted author manuscript

Link to publication record in Manchester Research Explorer

Citation for published version (APA):

Henderson, F., Hart, P. J., Pradillo, J. M., Kassiou, M., Christie, L., Williams, K. J., Boutin, H., \& McMahon, A. (2018). Multi-modal imaging of long-term recovery post-stroke by positron emission tomography and matrixassisted laser desorption/ionisation mass spectrometry. Rapid Communications in Mass Spectrometry, 32(9), 721 729. https://doi.org/10.1002/rcm.8090

\section{Published in:}

Rapid Communications in Mass Spectrometry

\section{Citing this paper}

Please note that where the full-text provided on Manchester Research Explorer is the Author Accepted Manuscript or Proof version this may differ from the final Published version. If citing, it is advised that you check and use the publisher's definitive version.

\section{General rights}

Copyright and moral rights for the publications made accessible in the Research Explorer are retained by the authors and/or other copyright owners and it is a condition of accessing publications that users recognise and abide by the legal requirements associated with these rights.

\section{Takedown policy}

If you believe that this document breaches copyright please refer to the University of Manchester's Takedown Procedures [http://man.ac.uk/04Y6Bo] or contact uml.scholarlycommunications@manchester.ac.uk providing relevant details, so we can investigate your claim.

\section{OPEN ACCESS}




\section{Multi-modal imaging of long term recovery post-stroke by PET and MALDI-MS}

Fiona Henderson ${ }^{1,2}$, Philippa J. Hart ${ }^{3}$, Jesus M. Pradillo ${ }^{4}$, Michael Kassiou ${ }^{5}$, Lidan Christie ${ }^{1}$ Kaye J. Williams ${ }^{2}$, Herve Boutin ${ }^{1}$, Adam McMahon ${ }^{1 *}$

1. Wolfson Molecular Imaging Centre, Division of Informatics, Imaging and Data Sciences, School of Health Sciences, Faculty of Biology, Medicine and Health, University of Manchester, Manchester Academic Health Science Centre, Manchester, $U K$

2. Division of Pharmacy and Optometry, School of Health Sciences, Faculty of Biology, Medicine and Health, University of Manchester, Manchester Academic Health Science Centre, Stopford Building, Manchester, UK

3. Shimadzu/Kratos Analytical, Trafford Wharf Road, Manchester M17 1GP

4. Unidad de Investigación Neurovascular, Departamento de Farmacología, Facultad de Medicina, Universidad Complutense (UCM) and Instituto de Investigación 12 de Octubre, Madrid, Spain

5. School of Chemistry, Australia \& Faculty of Health Sciences, University of Sydney, Sydney, NSW, 2006, Australia

\section{6.}

Correspondence to :

Adam McMahon, adam.mcmahon@manchester.ac.uk

Wolfson Molecular Imaging Centre, Division of Informatics, Imaging and Data Sciences, School of Health Sciences, Faculty of Biology, Medicine and Health, University of Manchester, Manchester Academic Health Science Centre, 27 Palatine Rd, Manchester, M20 $3 L J, U K$

This article has been accepted for publication and undergone full peer review but has not been through the copyediting, typesetting, pagination and proofreading process which may lead to differences between this version and the Version of Record. Please cite this article as doi: $10.1002 / \mathrm{rcm} .8090$ 


\section{Abstract}

RATIONALE: Stroke is a leading cause of disability worldwide. Understanding the recovery process post-stroke is essential; however, longer-term recovery studies are lacking. In-vivo Positron emission tomography (PET) can image biological recovery processes, but is limited by spatial resolution and its targeted nature. Untargeted mass spectrometry imaging offers high spatial resolution, providing an ideal ex-vivo tool for brain recovery imaging.

METHODS: Magnetic resonance imaging (MRI) was used to image a rat brain 48 hours after ischaemic stroke to locate the infarcted regions of the brain. Positron emission tomography (PET) was carried out three months post-stroke using the tracers $\left[{ }^{18} \mathrm{~F}\right] \mathrm{DPA}-714$ for TSPO and $\left[{ }^{18}\right.$ F]IAM6067 for sigma-1 receptors to image neuroinflammation and neurodegeneration respectively. The rat brain was flash-frozen immediately after PET scanning, and sectioned for matrix-assisted laser desorption ionisation mass spectrometry (MALDI-MS) imaging.

RESULTS: Three months after stroke, PET imaging shows minimal detection of neurodegeneration and neuroinflammation, indicating that the brain has stabilised. However, MALDI-MS images reveal distinct differences in lipid distributions, (e.g. phosphatidylcholine and sphingomyelin) between the scar and the healthy brain, suggesting that recovery processes are still in play. It is currently not known if the altered lipids in the scar will change on a longer time scale, or if they are stabilised products of the brain poststroke.

CONCLUSIONS: The data demonstrates the ability to combine MALD-MS with in vivo PET to image different aspects of stroke recovery. 


\section{Introduction}

Stroke is a leading cause of death and disability worldwide, with ischaemic stroke contributing towards $80 \%$ of all diagnosed strokes (1). Monitoring brain recovery post-stroke allows more effective and realistic rehabilitation of patients, and is also important for researching new treatments. However, there are few studies regarding the longer-term recovery after a stroke. The majority of brain recovery occurs within the first 30 days after a stroke, and many studies have focussed on this more immediate recovery of the brain $(2,3)$. However, recovery processes can continue for at least 6 months (3). Here, a multi-modal imaging approach is used to study recovery of an ischaemic stroke in a pre-clinical model, three months post-stroke.

During ischaemic stroke, there is a lack of oxygen and nutrients delivered to an area of the brain, and an infarct (an area of dead tissue) is formed. The tissue surrounding the infarct is moderately ischaemic but can survive for a short period of time, and is known as the penumbra. However, within days cells in the penumbra will also die, and the penumbra will become part of the infarct. This loss of tissue and neurons in the infarct will then be responsible for the final loss of functionality. Furthermore, remote areas which are connected to the infarct can also be affected.

Imaging techniques are ideal for studying the brain post-stroke, with conventional methods of diagnosing a stroke including computed tomography (CT) and magnetic resonance imaging (MRI). CT is preferred as it is cheaper and quicker, although MRI is much more sensitive in detecting acute ischaemic stroke (4). Both CT and MRI are well-established clinical imaging tools and they can show where the infarct has occurred, and if thrombolysis is necessary and feasible (4-6). MRI is an important imaging modality used to monitor the brain acutely after a stroke; however other imaging modalities such as functional MRI (fMRI) or PET are preferential for imaging long-term recovery, as they are able to image functionality in the brain.

fMRI measures blood flow which is coupled to brain activity, and so can detect functional activity in the brain as oxygen is delivered from the blood to activated brain regions. Studies have shown that fMRI is a useful imaging tool for monitoring recovery post-stroke $(7,8)$ with Loubinoux et al showing that fMRI maps give a better indication of behavioural outcome 
than total infarct volume as determined by MRI (9). PET is a highly sensitive technique where a radiolabelled molecule (tracer) is imaged in-vivo. As well as measuring blood flow, it can give information on other important aspects of stroke pathology, including inflammation (10). Neuroinflammation and neurodegeneration are key markers of pathology post-stroke, with neurodegeneration being the loss of neuronal integrity, which can include loss of function and structure. Numerous PET tracers have been developed to image neuroinflammation through expression of the translocator protein $18 \mathrm{kDa}$ (TSPO) by glial cells and infiltrating macrophages(11-13). There is, however, a lack of reliable and broadly accepted biomarkers to image neurodegeneration with PET. The sigma-1 receptor (S1R) has been suggested as a potential pan-neuronal biomarker suitable for PET imaging, and several tracers have been developed for this purpose $(14,15)$.

The S1R is a neuronal biomarker, which is involved in the regulation of intracellular $\mathrm{Ca}^{2+}$ signalling (16). A stroke can cause a disruption of calcium signalling, resulting in neuronal cell death (17). Here, TSPO and S1R tracers are used at three months post-stroke to monitor neuroinflammation and neurodegeneration in long-term recovery.

One drawback of PET is that it can only image one tracer at a time, and is therefore very much a targeted technique. Moreover, by using pre-determined tracers, PET is very much a hypothesis driven imaging technique. Conversely, mass-spectrometry imaging can investigate a large number of molecules at once without a priori knowledge. Matrix-assisted laser desorption ionisation mass spectrometry (MALDI-MS) imaging can image a large number of both exogenous and endogenous species in one experiment, and at very high spatial resolutions (10-100 $\mu \mathrm{m})$. MALDI-MS has been used in previous studies to image the acute effects of lipid distributions post-stroke (18), showing that lipid alterations are prominent shortly after stroke. Phospholipids play both a structural and a signalling role in the body, and are of particular abundance in the brain. These phospholipids undergo changes after a traumatic brain injury such as stroke, with phospholipid degradation being recognised as a promoter of neuronal death (19). Furthermore, lipids are involved in inflammation and cell death (20-22). Thus, MALDI-MS lipid imaging data can enrich the in-vivo PET data regarding neuroinflammation and neurodegeneration, and they have been used so to study brain recovery three months post-stroke. 


\section{Methods}

\section{Materials}

All solvents were purchased from Sigma-Aldrich, Gillingham, UK

Phosphate buffered saline (PBS) was made using tablets purchased from Oxoid, Basingstoke, UK that were dissolved in distilled $\mathrm{H}_{2} \mathrm{O}$.

$1 \mathrm{~mL}$ Tween (T) (Fisher Scientific, Loughborough, UK) was added to PBS to make PBS-T. Bovine serum albumin (BSA) (Sigma-Aldrich) was dissolved at $1 \mathrm{mg} / \mathrm{mL}$ PBST to make PBST-BSA solution.

\section{Induction of focal cerebral ischaemia in rats}

Wistar rats (350g, Charles River, Margate, UK) were allowed free access to food and water and were maintained under temperature, humidity, and light-controlled conditions. All procedures were performed under appropriate UK Home Office licences and adhered to the UK Animals (Scientific Procedures) Act (1986).

Middle cerebral artery occlusion (MCAO) was induced by 70 min transient ligature of the left middle cerebral artery trunk and common carotid artery with a 10-0 suture (Prolene, Ethicon, Somerville, NJ, USA) as described previously (23) under anaesthesia using isoflurane (2-3\% for induction and 1.5-2\% during surgery) in a mixture of $70 \% \mathrm{~N}_{2} \mathrm{O}$ and $30 \% \mathrm{O}_{2}$. Core body temperature was maintained at $37.0 \pm 0.5^{\circ} \mathrm{C}$ throughout the surgery by a heating blanket (Homeothermic Blanket Control Unit; Harvard Apparatus, Edenbridge, UK). Occlusion and reperfusion were confirmed visually under the surgical microscope. After surgery, animals were returned to their home cages and allowed free access to water and food.

\section{$M R I$}

MCAO lesions were assessed by $\mathrm{T} 2$ weighted $\left(\mathrm{T}_{2} \mathrm{~W}\right) \mathrm{MRI}$ at $48 \mathrm{~h}$ after stroke, using a 7-T, horizontal-bore magnet (Agilent Technologies, Cheadle, UK) interfaced to a Bruker Avance III console (Bruker Biospin, Coventry, UK) using a surface transmit-receive coil, as described previously (24). Images were visualised using Anatomist software (http://brainvisa. 
info), and an anatomical atlas as described in (25) was used to identify different brain regions.

\section{PET}

The two PET scans were acquired 5 days apart three months post-stroke.

N-(2-benzofuranylmethyl)-N'-[4-(2-fluoroethoxy)benzyl]piperazine (IAM6067) (S1R tracer) was labelled with $\left[{ }^{18} \mathrm{~F}\right]$ as previously described by Moussa et al. (15) $36 \mathrm{MBq}$ in $260 \mu \mathrm{L}$ of saline $\left(0.395 \mathrm{nmol}\right.$ at $94 \mathrm{GBq} / \mu \mathrm{mol}, 100 \%$ radiochemical purity) of $\left[{ }^{18} \mathrm{~F}\right]-\mathrm{IAM} 6067$ was injected intravenously at the start of the scan.

$\left[{ }^{18} \mathrm{~F}\right]-N, N$-diethyl-2-[4-(2-fluoroethoxy)phenyl]-5,7-dimethylpyrazolo[1,5- $\left.a\right]$ pyrimidine-3acetamide ([ ${ }^{18}$ F]DPA-714) (TSPO tracer) was synthesised as described elsewhere $(24,26)$. Briefly, $\left[{ }^{18} \mathrm{~F}\right] \mathrm{DPA}-714$ underwent nucleophilic aliphatic substitution of a tosylate precursor (26). $29 \mathrm{MBq}$ in $483 \mu \mathrm{L}$ of saline $(0.898 \mathrm{nmol}$ at $32 \mathrm{GBq} / \mu \mathrm{mol}, 100 \%$ radiochemical purity) of $\left[{ }^{18}\right.$ F]DPA-714 was injected intravenously at the start of the scan.

PET scans were performed on a Siemens Inveon ${ }^{\circledR}$ PET/CT scanner (Siemens Healthcare Ltd, Camberley, UK) for 60 minutes. The rat was anesthetized using isoflurane (induction 5\%, 2$2.5 \%)$ and catheterized in the tail vein for tracer injection. Animal respiration and temperature were controlled using a pressure-sensitive pad and rectal probe (BioVet, $\mathrm{m} 2 \mathrm{~m}$ Imaging Corp., Cleveland, $\mathrm{OH}$, USA) and the body temperature was maintained at $37 \pm 0.7^{\circ} \mathrm{C}$ via the interface managed by the BioVet system. PET-CT was performed as previously described (24). Briefly, a CT scan was performed prior to the PET acquisition for attenuation correction. The list mode emission data were histogrammed into 3D sinograms of 35 dynamic frames, normalized, corrected for dead-time, attenuation, scatter and radioactive decay, and reconstructed using OSEM3D (16 subsets and 4 iterations) into images of dimensions $128 \times 128 \times 159$ voxels of volume $0.776 \times 0.776 \times 0.796 \mathrm{~mm}^{3}$.

\section{Sample preparation}

After PET scanning, rats were promptly decapitated, and their brains removed and frozen in isopentane. Brains were stored at $-80^{\circ} \mathrm{C}$. Brains were sectioned to $12 \mu \mathrm{m}$ and thaw-mounted 
onto indium tin oxide (ITO) slides (Sigma-Aldrich). Sections were washed based on a protocol modified from Wang et al (27) where $100 \mu \mathrm{L}$ of $150 \mathrm{mM}$ ammonium acetate (Sigma-Aldrich) was pipetted over the sections, and allowed to dry between washes. Sections were washed 10 times. Slides were sprayed with 10mg/ml 2,5-dihydroxybenzoic acid (DHB) (LaserBio Labs, Sophia-Antipolis, France) made up in 70:30 methanol:water and 0.1\% trifluoroacetic acid (TFA), (Sigma-Aldrich) using the SunCollect ${ }^{\mathrm{TM}}$ (Sunchrom, Friedrichsdorf, Germany). Flow rate of $10 \mu \mathrm{L} / \mathrm{min}, 15 \mu \mathrm{L} / \mathrm{min}$ and $20 \mu \mathrm{L}$ weres used for the first, second and third layers, respectively, followed by 27 layers at $25 \mu \mathrm{L} / \mathrm{min}$.

\section{MALDI-MS imaging}

Imaging was performed on a MALDI-7090 TOF-TOF mass spectrometer (Shimadzu, Manchester, UK), equipped with a solid-state UV laser. Experiments were performed in positive ion reflectron mode, using 100 laser shots per profile and a laser beam diameter of $50 \mu \mathrm{m}$ at the sample. The laser repetition rate was $500 \mathrm{~Hz}$, and the spatial resolution was set to $80 \mu \mathrm{m}$. The blanking mass was set to $\mathrm{m} / \mathrm{z}, 50$ and pulsed extraction to $\mathrm{m} / \mathrm{z} 700$. Images were normalised to the total ion current (TIC) using a Shimdzu MALDI imaging processing toolbox beta version and processed in BioMap (Novartis, Basel, Switzerland). Slides were washed with $70 \%$ ethanol post imaging and stained with haematoxylin and eosin (H\&E). The images were compared with those in an anatomical atlas as described elsewhere (25).

\section{MALDI MS/MS}

MALDI MS/MS experiments were performed on the MALDI-7090 mass spectrometer using collision induced dissociation (CID) (helium was used as the CID gas) and axial spatial distribution focussing (ASDF) (28). Interpretation of the spectra was facilitated by using the LIPID MAPS Lipidomics Gateway database (http://www.lipidmaps.org) (29).

\section{$H \& E$}

H\&E staining was carried out on the same sections as the MALDI-MS imaging. Slides with mounted sections were immersed in xylene for two minutes, and then transferred to industrial 
methylated spirit for 4 × 2 minutes. Sections were then immersed in tap water followed by 3 minutes in Harris haematoxylin (Sigma-Aldrich), a rinse in hot water, and a one second dip in eosin (Leica, Milton Keynes, UK). Slides were transferred to industrial methylated spirit for ( 4 x 2 minutes), then to xylene ( 4 × 2 minutes), and left to dry. Aqueous mounting media (Vector labs, Burlingame, CA, USA) were used to mount coverslips (VWR, Lutterworth, UK) onto slides, which were left overnight to dry. Images were acquired using a [20x/0.80 Plan Apo] objective using the 3D Histech Pannoramic 250 Flash II slide scanner, and processed using Pannoramic viewer (3D HISTECH, Budapest, Hungary).

\section{Immunofluorescence}

Tissue sections were fixed in ice-cold acetone (10 minutes). After drying, a DAKO pen (Agilent Technologies) was used to draw around each of the sections, and blocking solution was added to each section (10\% goat serum in PBST). Slides were incubated at room temperature for 15 mins and then washed ( 2 x 3 mins) in PBST-BSA. Anti-glial fibrillary acidic protein (rabbit, Agilent Technologies) at 1:1000 was made up in PBST-BSA, and 10\% normal goat serum. The primary antibody solution was left on tissue sections at $4{ }^{\circ} \mathrm{C}$ overnight. Sections were washed (3 x 4 minutes) in PBS. A secondary antibody solution containing 1:150 of Cy5 goat anti-Rb (Invitrogen, Paisley, UK) was prepared in PBST-BSA; and pipetted onto each section and left for $60 \mathrm{mins}$ in the dark at room temperature. Slides were washed again with PBS ( 3 x 4 minutes). Fluorescent mounting media (Abcam, Cambridge, UK) were used to mount coverslips (VWR) onto slides. Images were acquired using a [20x/0.80 Plan Apo] objective using the 3D Histech Pannoramic 250 Flash II slide scanner, and processed using the Panoramic viewer (3D HISTECH).

\section{Results and Discussion}

MRI is an established technique for imaging the brain acutely after stroke. Here, MRI was carried out 48 hours post-ischaemic stroke, and shows a distinct infarct region in the right hemisphere (Figure 1), revealing where the ischaemia has occurred.

Three months post-stroke, PET detects no substantial neuroinflammation in the peri-infarct area (Figure 2). We have previously shown that $\left[{ }^{18} \mathrm{~F}\right]$-DPA-714 can be used as a PET tracer 
to image TSPO post-ischaemia (30); however, these scans were carried out less than a week post-stroke. Neuroinflammation is expected to be at its most aggressive in the first two weeks after stroke (31-33), with Martin et al showing a peak of TSPO expression at day 11, using $\left[{ }^{18} \mathrm{~F}\right]-\mathrm{DPA}-714$ PET. Few imaging studies have been carried out to investigate chronic inflammation post-stroke, but previous studies analysing post-mortem tissue suggest that inflammation should dissipate after three months $(34,35)$. Walberer et al showed by PET that at day 42, microglial activation was still present, but it had moved away from the infarct region to the thalamus (36). Here, PET scans showed that there was no defined TSPO overexpression relating to the brain anatomy or penumbra.

PET scans do not reveal any decrease in S1R in the infarct or the peri-infarct area compared with the rest of the brain (Figure 2), suggesting remodelling or reorganisation of the brain structure around the initial infarct. The absence of a decrease in the S1R tracer $\left[{ }^{18} \mathrm{~F}\right]$ IAM6067 in the area of the initial infarct is probably due to the deformation of the brain, where dead brain tissue has collapsed into the infarct and been replaced by a thin astrocytic scar (Figures S1 and S2, supporting information) and healthy tissue from the former peri-infarct tissue. This healthy tissue, along with the low resolution of PET, would explain why the loss of neurons that had occurred acutely in the infarct is no longer visible three months after stroke. Consequently, the $\left[{ }^{18} \mathrm{~F}\right] \mathrm{IAM} 6067$ PET scans, taken together with the lack of inflammation observed by $\left[{ }^{18} \mathrm{~F}\right]-\mathrm{DPA}-714$ PET scans, suggest that the brain has stabilised post-stroke.

Lipids play crucial roles in membrane formation, cell death, and cell signalling; and thus will be important in neurodegeneration and inflammation processes. Ex-vivo MALDI-MS imaging was carried out after PET scanning to investigate lipid changes in stroke recovery. Matrix effects in brain MALDI-MS images due to altered levels of sodium in the infarct post-stroke has been discussed by Lankeoff et al (37), and therefore care should be taken when investigating sodiated lipid peaks in the infarct . $\mathrm{Na}^{+}$levels are expected to rise inside the cell acutely post-stroke due to the cessation of $\mathrm{Na}^{+} / \mathrm{K}^{+}$ATPase activity. However, because this study was carried out three months after ischaemia, the difference in sodium concentration across the brain is not expected to be a significant issue. Furthermore, Figure S3 (supporting information) shows an example of protonated and sodiated lipid species localising in the same area.

Unlike the PET data, MALDI-MS imaging showed clear molecular differences between the scar region, and the rest of the brain (Figure 3). The different lipid profile of the scar from 
that of normal brain tissue indicates that lipid metabolism is still altered in the brain three months after the ischaemic attack.

During an ischaemic attack there is an increase in the release of neurotransmitter glutamate, which causes intracellular $\mathrm{Ca}^{2+}$ to rise, and phospholipases (A2, C, D) to be activated (38, 39). The cytokines TNF- $\alpha$ and interleukin-1 upregulated during the pro-inflammatory response acutely after an ischaemic attack will also cause activation of phospholipases (40, 41). Phospholipase A2 ( $\left.\mathrm{PLA}_{2}\right)$ hydrolyses phospholipids into lysophospholipids, which in turn can act as cell signalling molecules. The upregulation of PLA2 in the ischaemic brain thus causes an upregulation of lysophospholipids and downregulation of phospholipids in the infarct region acutely after stroke $(42,43)$. Phosphatidylcholines (PCs) are a class of phospholipids that are major cell membrane components. It is interesting that some PC species, including PC (16:0/18:1) and PC (16:0/16:0) (MS/MS shown in Figures S4 and S5, supporting information), appear across the healthy brain tissue (Figure 4), but are clearly absent in the scar region three months after stroke. A previous study on the acute effects of stroke showed that PC (16:0/18:1) is downregulated in the infarct (44). This study reveals that downregulation of PC (16:0/18:1) and PC (16:0/16:0) in the scar is a long-term effect after an ischaemic stroke. TSPO PET scans indicate no neuroinflammation in the scar region of the brain three months post-stroke, suggesting that chronic differences in PC and LPC expression are no longer a result of cytokine action.

In addition to phospholipase activation, the cytokines TNF- $\alpha$ and interleukin- 1 cause an increase in sphingomyelin (SM) hydrolysis via sphingomyelinase activity. The hydrolysis of SM leads to the release of ceramides causing apoptosis (reviewed in (45)), and this mechanism is prevalent during a stroke. Upregulation of SM (d18:1/16:0) is seen three months post-stroke (Figure 5) (MS/MS results shown in Figure S6, supporting information), indicating that sphingomyelinase activity has ceased and inflammation (via TNF- $\alpha$ and interleukin-1) has diminished. Roux et al showed that SM (d18:1/16:0) was also upregulated in the injured region of a rat brain 7 days post traumatic brain injury (46), and so in combination with the data presented here, this suggests that SM (d18:1/16:0) upregulation is sustained as a long-term effect of brain injury. SM (d18:1/16:0) is also present in the lateral ventricles, so it is feasible that leakage of cerebral spinal fluid into the scar region is the cause of the upregulation of the SM three months post-stroke. 
The abundance of SM (d18:1/16:0) in the scar region suggests a decrease in neuroinflammation. This is in agreement with the TSPO PET scan (Figure 2), which shows no neuroinflammation in the rat brain three months after an ischaemic attack.

Lysophosphatidylcholine (LPC) (16:0) (MS/MS shown in Figure S7 (supporting information) is present at higher abundance in the scar region (Figure 5) than in the rest of the brain. It has already been established that PC species with 16:0 fatty acid chains are absent in the scar region (Figure 4), indicative of high rates of $\mathrm{PLA}_{2}$ in the scar region, which is converting PC species to LPC (16:0). Koizumi et al found upregulation of LPC (16:0) in the infarct region 24hrs post-stroke (44). Interestingly, this feature remains in the ischaemic brain three months after stroke. LPCs are associated with a pro-inflammatory response, and studies indicate the involvement of LPCs in microglia activation (LPC can stimulate interleukin-1 $\beta$ production by microglia) (21) (20). However, TSPO PET scans indicate no increase in inflammation in the scar region. TSPO PET targets mostly activated microglia; however, the relationship between TSPO expression and microglia/astrocyte phenotypes still remains unclear. TSPO is a biomarker of microglia activation and proliferation in the sub-acute (days) following stroke (13); however, more complex processes continue to happen in glial cells long after stroke which are likely to be uncorrelated with TSPO expression, and LPC 16:0 may be playing a role in these. Furthermore, LPC 16:0 is also known to be a second messenger for a number of cascades (e.g. protein kinase $\mathrm{C}$ and phospholipases) $(47,48)$ and so this work suggests a more complex role of LPC 16:0 than its involvement in microglial activation.

A peak was detected at $m / z 806.5$ which localised in a subset of the scar region in Section 1 , and also across the whole brain, including the healthy isocortex (Figure 6). The peak at $\mathrm{m} / \mathrm{z}$ 806.5 was not identified but is hypothesised to be a phospholipid species based on comparison with the LIPID MAPS database (29). This finding suggests that the lipidome in part of the scar region has stabilised, in line with PET data showing stabilisation of neuroinflammation and neuronal cell death. However, the hypothesised phospholipid peaks at $\mathrm{m} / \mathrm{z} 824.5$ and 768.5 , which also appear in the scar (as well as healthy brain regions), interestingly show localisations in a different subset of the scar region from $m / z$ 806. These lipid species could be playing a role in long-term recovery and repair. Unfortunately, the peaks at $\mathrm{m} / z, 768$ and $\mathrm{m} / \mathrm{z}, 824$ could not be identified, although $\mathrm{m} / \mathrm{z}, 824$ has previously been seen in the brain by MALDI-MS, and was identified as a potassiated PC 36:2 (49). Wieloch et al reviewed brain recovery processes after stroke, and stated that at three months, inflammation had diminished but neurogenesis and gliogenesis were still prevalent (50). The 
peaks at $m / z 824$ and $m / z 768$ are probably membrane lipid species, and thus could be playing an important role in the formation of new glial cells or neurons.

\section{Conclusion}

MRI, PET and MALDI-MS have been used in this study as investigatory techniques to image different aspects of brain recovery post-stroke. MRI is ideal for visualising the infarct shortly after stroke, whereas PET and MALDI-MS can give information on biological mechanisms occurring in the long-term recovery from stroke. MALDI-MS imaging of lipids in the brain allows imaging of recovery after ischaemic stroke for molecules that are thus far inaccessible to PET or other in vivo modalities. Furthermore, MALDI-MS imaging was able to reveal (lipid) biomarkers associated with the scar ex-vivo, which were not revealed using the tracers in this study. The exact biochemical roles of the lipid biomarkers revealed by MALDI-MS remain to be elucidated.

\section{Acknowledgements}

The Bioimaging Facility microscopes used in this study were purchased with grants from BBSRC, Wellcome and the University of Manchester Strategic Fund. Special thanks go to Roger Meadows for his help with the microscopy and image analysis. This research received support from the European Union's Seventh Framework Programme (FP7/2007-2013) under grant agreement ${ }^{\circ}$ HEALTH-F2-2011-278850 (INMiND).

Thanks go to Matthew Openshaw and Kratos Analytical Ltd for instrument time and technical support.

\section{Author contributions}

FH and PJH carried out MALDI-MS imaging sample preparation (washing, matrix application), and MALDI-MS imaging. In addition, FH performed the MALDI-MS image analysis, MALDI-MS/MS, lipid identification, and the H\&E and immunofluorescence staining. 
JMP developed the MCAO model, and MK developed the TSPO tracer used in this study. LC sectioned the brain tissue, and assisted HB in the induction of the focal cerebral ischaemia, and PET and MRI scanning. HB carried out PET and MRI image analysis. KJW and AM supervised $\mathrm{FH}$ and provided advice and guidance during this research.

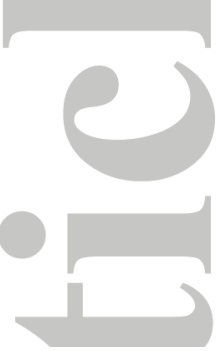

\section{References}

1. Lloyd-Jones D, Adams RJ, Brown TM, et al. Executive summary: heart disease and stroke statistics--2010 update: a report from the American Heart Association. Circulation. 2010;121(7):948-54.

2. Skilbeck CE, Wade DT, Hewer RL, Wood VA. Recovery after stroke. J Neurol Neurosurg Psychiatry. 1983;46(1):5-8.

3. Duncan PW, Lai SM, Keighley J. Defining post-stroke recovery: implications for design and interpretation of drug trials. Neuropharmacology. 2000;39(5):835-41.

4. Chalela JA, Kidwell CS, Nentwich LM, et al. Magnetic resonance imaging and computed tomography in emergency assessment of patients with suspected acute stroke: a prospective comparison. Lancet. 2007;369(9558):293-8.

5. Ciumas C, Montavont A, Ryvlin P. Magnetic resonance imaging in clinical trials. Curr Opin Neurol. 2008;21(4):431-6.

6. Rajajee V, Kidwell C, Starkman S, et al. Diagnosis of lacunar infarcts within 6 hours of onset by clinical and CT criteria versus MRI. J Neuroimaging. 2008;18(1):66-72.

7. Brodtmann A, Puce A, Darby D, Donnan G. Serial functional imaging poststroke reveals visual cortex reorganization. Neurorehabil Neural Repair. 2009;23(2):150-9.

8. Askim T, Indredavik B, Vangberg T, Haberg A. Motor network changes associated with successful motor skill relearning after acute ischemic stroke: a longitudinal functional magnetic resonance imaging study. Neurorehabil Neural Repair. 2009;23(3):295-304.

9. Loubinoux I, Dechaumont-Palacin S, Castel-Lacanal E, et al. Prognostic value of FMRI in recovery of hand function in subcortical stroke patients. Cereb Cortex. 2007;17(12):2980-7. 
10. Drake C, Boutin H, Jones MS, et al. Brain inflammation is induced by co-morbidities and risk factors for stroke. Brain Behavior and Immunity. 2011;25(6):1113-22.

11. Weinstein JR, Koerner IP, Moller T. Microglia in ischemic brain injury. Future Neurol. 2010;5(2):227-46.

12. Rupprecht R, Papadopoulos V, Rammes G, et al. Translocator protein (18 kDa) (TSPO) as a therapeutic target for neurological and psychiatric disorders. Nat Rev Drug Discov. 2010;9(12):971-88.

13. Boutin H, Pinbor LH. TSPO imaging in stroke: from animal models to human subjects. Clinical and Translational Imaging. 2015;3(6):423-35.

14. James ML, Shen B, Nielsen $\mathrm{CH}$, et al. Evaluation of sigma-1 receptor radioligand 18F-FTC-146 in rats and squirrel monkeys using PET. J Nucl Med. 2014;55(1):147-53.

15. Moussa IA, Banister SD, Giboureau N, Meikle SR, Kassiou M. Synthesis and in vivo evaluation of [18F]N-(2-benzofuranylmethyl)-N'-[4-(2-fluoroethoxy)benzyl]piperazine, a novel sigma1 receptor PET imaging agent. Bioorg Med Chem Lett. 2011;21(22):6820-3.

16. Hayashi T. Sigma-1 receptor: the novel intracellular target of neuropsychotherapeutic drugs. J Pharmacol Sci. 2015;127(1):2-5.

17. Mattson MP. Apoptosis in neurodegenerative disorders. Nat Rev Mol Cell Biol. 2000;1(2):120-9.

18. Hankin JA, Farias SE, Barkley RM, et al. MALDI mass spectrometric imaging of lipids in rat brain injury models. J Amn Soc Mass Spectrom. 2011;22(6):1014-21.

19. Adibhatla RM, Hatcher JF, Dempsey RJ. Phospholipase A2, hydroxyl radicals, and lipid peroxidation in transient cerebral ischemia. Antioxid Redox Signal. 2003;5(5):647-54.

20. Stock C, Schilling T, Schwab A, Eder C. Lysophosphatidylcholine stimulates IL1beta release from microglia via a P2X7 receptor-independent mechanism. J Immunol. 2006;177(12):8560-8.

21. Schilling T, Lehmann F, Ruckert B, Eder C. Physiological mechanisms of lysophosphatidylcholine-induced de-ramification of murine microglia. $J$ Physiol. 2004;557(Pt 1):105-20.

22. Kim HY. Phospholipids: a neuroinflammation emerging target. Nat Chem Biol. 2015;11(2):99-100.

23. Pradillo JM, Murray KN, Coutts GA, et al. Reparative effects of interleukin-1 receptor antagonist in young and aged/co-morbid rodents after cerebral ischemia. Brain Behav Immun. 2016. 
24. Boutin H, Murray K, Pradillo J, et al. 18F-GE-180: a novel TSPO radiotracer compared to 11C-R-PK11195 in a preclinical model of stroke. Eur J Nucl Med Mol Imaging. 2015;42(3):503-11.

25. Schwarz AJ, Danckaert, A, Reese T, et al. A stereotaxic MRI template set for the rat brain with tissue class distribution maps and co-registered anatomical atlas: Application to pharmacological MRI. Neuroimage. 2006(32):538-50.

26. James ML, Fulton RR, Vercoullie J, et al. DPA-714, a new translocator proteinspecific ligand: Synthesis, radiofluorination, and pharmacologic characterization. Journal of Nuclear Medicine. 2008;49(5):814-22.

27. Wang HY, Liu CB, Wu HW. A simple desalting method for direct MALDI mass spectrometry profiling of tissue lipids. J Lipid Res. 2011;52(4):840-9.

28. Belgacem O, Pittenauer E, Openshaw ME, Hart PJ, Bowdler A, Allmaier G. Axial spatial distribution focusing: improving MALDI-TOF/RTOF mass spectrometric performance for high-energy collision-induced dissociation of biomolecules. Rapid Commun Mass Spectrom. 2016;30(3):343-51.

29. Sud M, Fahy E, Cotter D, et al. LMSD: LIPID MAPS structure database. Nucleic Acids Res. 2007;35:D527-D32.

30. Boutin H, Prenant C, Maroy R, et al. [18F]DPA-714: direct comparison with [11C]PK11195 in a model of cerebral ischemia in rats. PLoS One. 2013;8(2):e56441.

31. Toth M, Little P, Arnberg F, et al. Acute neuroinflammation in a clinically relevant focal cortical ischemic stroke model in rat: longitudinal positron emission tomography and immunofluorescent tracking. Brain Struct Funct. 2016;221(3):1279-90.

32. Martin A, Boisgard R, Kassiou M, Dolle F, Tavitian B. Reduced PBR/TSPO

Expression After Minocycline Treatment in a Rat Model of Focal Cerebral Ischemia: A PET Study Using [F-18]DPA-714. Mol Imaging Biol. 2011;13(1):10-5.

33. Martin A, Boisgard R, Theze B, et al. Evaluation of the PBR/TSPO radioligand [F18]DPA-714 in a rat model of focal cerebral ischemia. J Cerebr Blood F Met. 2010;30(1):230-41.

34. Lehrmann E, Christensen T, Zimmer J, Diemer NH, Finsen B. Microglial and macrophage reactions mark progressive changes and define the penumbra in the rat neocortex and striatum after transient middle cerebral artery occlusion. J Comp Neurol. 1997;386(3):461-76. 


\section{Nowicka D, Rogozinska K, Aleksy M, Witte OW, Skangiel-Kramska J.}

Spatiotemporal dynamics of astroglial and microglial responses after photothrombotic stroke in the rat brain. Acta Neurobiol Exp (Wars). 2008;68(2):155-68.

36. Walberer M, Jantzen SU, Backes H, et al. In-vivo detection of inflammation and neurodegeneration in the chronic phase after permanent embolic stroke in rats. Brain Res. 2014;1581:80-8.

37. Lanekoff I, Stevens SL, Stenzel-Poore MP, Laskin J. Matrix effects in biological mass spectrometry imaging: identification and compensation. Analyst. 2014;139(14):3528-32.

38. Lipton P. Ischemic cell death in brain neurons. Physiol Rev. 1999;79(4):1431-568.

39. Siesjo BK. Pathophysiology and treatment of focal cerebral ischemia. Part II:

Mechanisms of damage and treatment. J Neurosurg. 1992;77(3):337-54.

40. Loddick SA, Rothwell NJ. Neuroprotective effects of human recombinant interleukin1 receptor antagonist in focal cerebral ischaemia in the rat. J Cereb Blood Flow Metab. 1996;16(5):932-40.

41. Liu T, Clark RK, McDonnell PC, Young PR, White RF, Barone FC, et al. Tumor necrosis factor-alpha expression in ischemic neurons. Stroke. 1994;25(7):1481-8.

42. Phillis JW, O'Regan MH. The role of phospholipases, cyclooxygenases, and lipoxygenases in cerebral ischemic/traumatic injuries. Crit Rev Neurobiol. 2003;15(1):61-90. 43. Adibhatla RM, Hatcher JF, Larsen EC, Chen X, Sun D, Tsao FH. CDP-choline significantly restores phosphatidylcholine levels by differentially affecting phospholipase A2 and CTP: phosphocholine cytidylyltransferase after stroke. J Biol Chem. 2006;281(10):671825.

44. Koizumi S, Yamamoto S, Hayasaka T, et al. Imaging mass spectrometry revealed the production of lyso-phosphatidylcholine in the injured ischemic rat brain. Neuroscience. 2010;168(1):219-25.

45. Pettus BJ, Chalfant CE, Hannun YA. Ceramide in apoptosis: an overview and current perspectives. Biochim Biophys Acta. 2002;1585(2-3):114-25.

46. Roux A, Muller L, Jackson SN, et al. Mass spectrometry imaging of rat brain lipid profile changes over time following traumatic brain injury. J Neurosci Meth. 2016;272:19-32. 47. Prokazova NV, Zvezdina ND, Korotaeva AA. Effect of lysophosphatidylcholine on transmembrane signal transduction. Biochemistry (Mosc). 1998;63(1):31-7.

48. Okajima F, Sato K, Tomura H, et al. Stimulatory and inhibitory actions of lysophosphatidylcholine, depending on its fatty acid residue, on the phospholipase $\mathrm{C} / \mathrm{Ca} 2+$ system in HL-60 leukaemia cells. Biochem J. 1998;336 ( Pt 2):491-500. 
49. Matsumoto J, Sugiura Y, Yuki D, et al. Abnormal phospholipids distribution in the prefrontal cortex from a patient with schizophrenia revealed by matrix-assisted laser desorption/ionization imaging mass spectrometry. Anal Bioanal Chem. 2011;400(7):1933-43. 50. Wieloch T, Nikolich K. Mechanisms of neural plasticity following brain injury. Curr Opin Neurobiol. 2006;16(3):258-64.

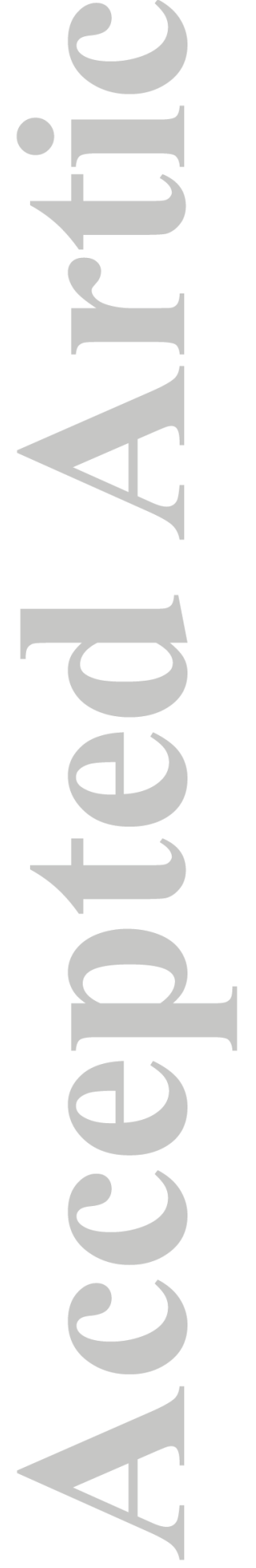




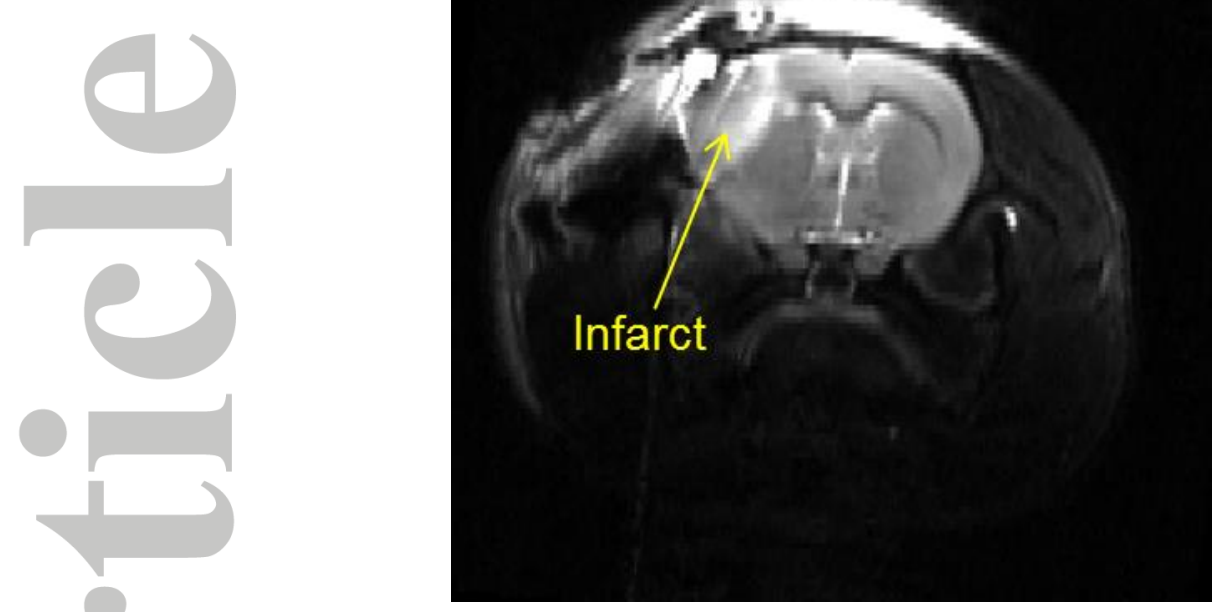

Fig.1. MRI scan showing the infarct in the right hemisphere of a rat brain 48 hours post stroke.
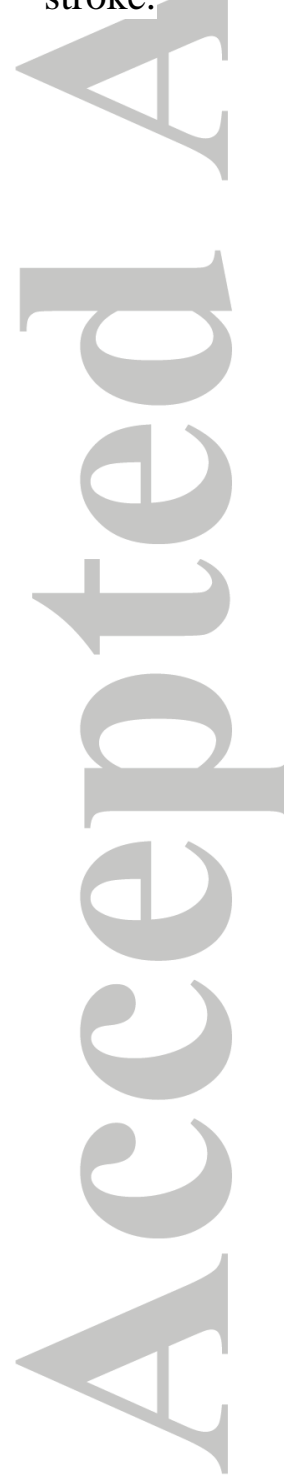

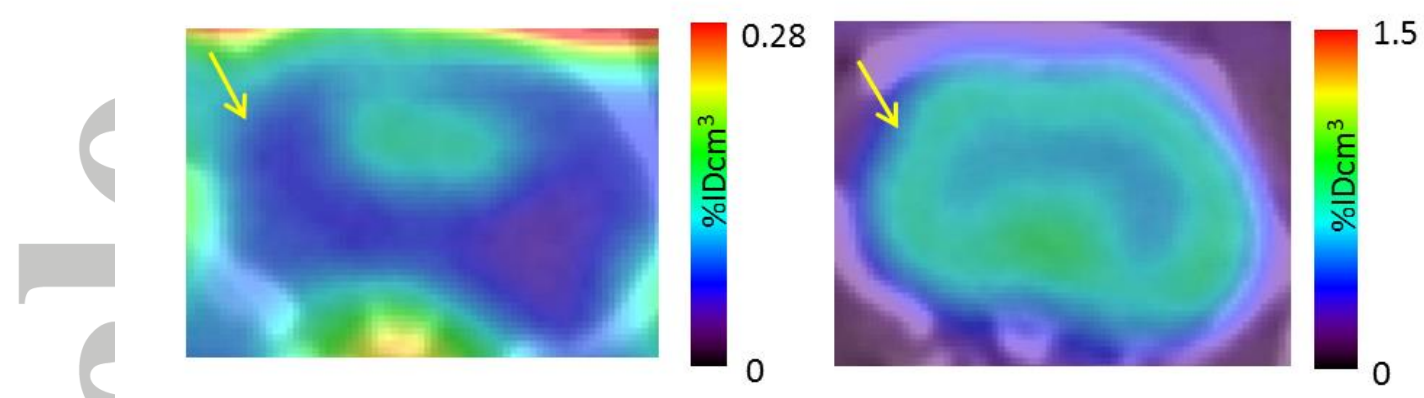

Fig.2. $\left[{ }^{18} \mathrm{~F}\right]-\mathrm{DPA}-714$ (left) and IAM6067 (right) PET scans of a rat brain three months postischaemia, showing TSPO and S1R receptors respectively. Arrows have been drawn to indicate the area of the original infarct.
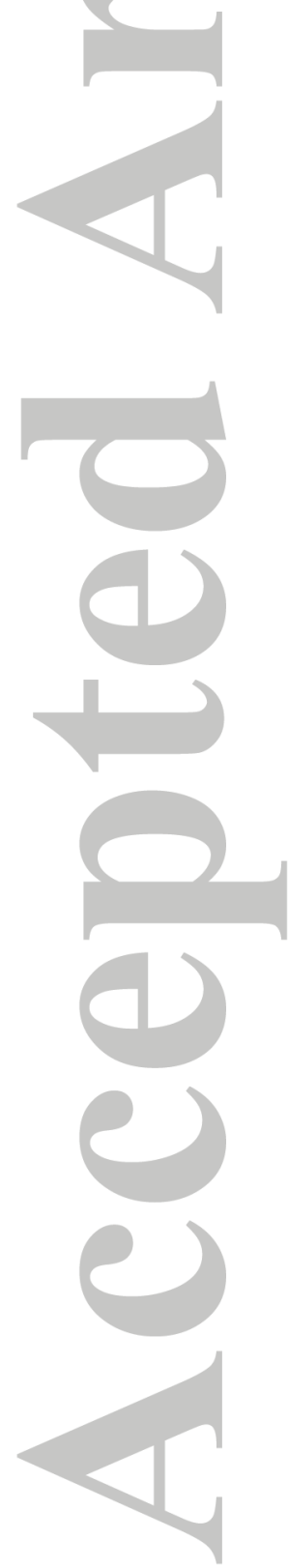


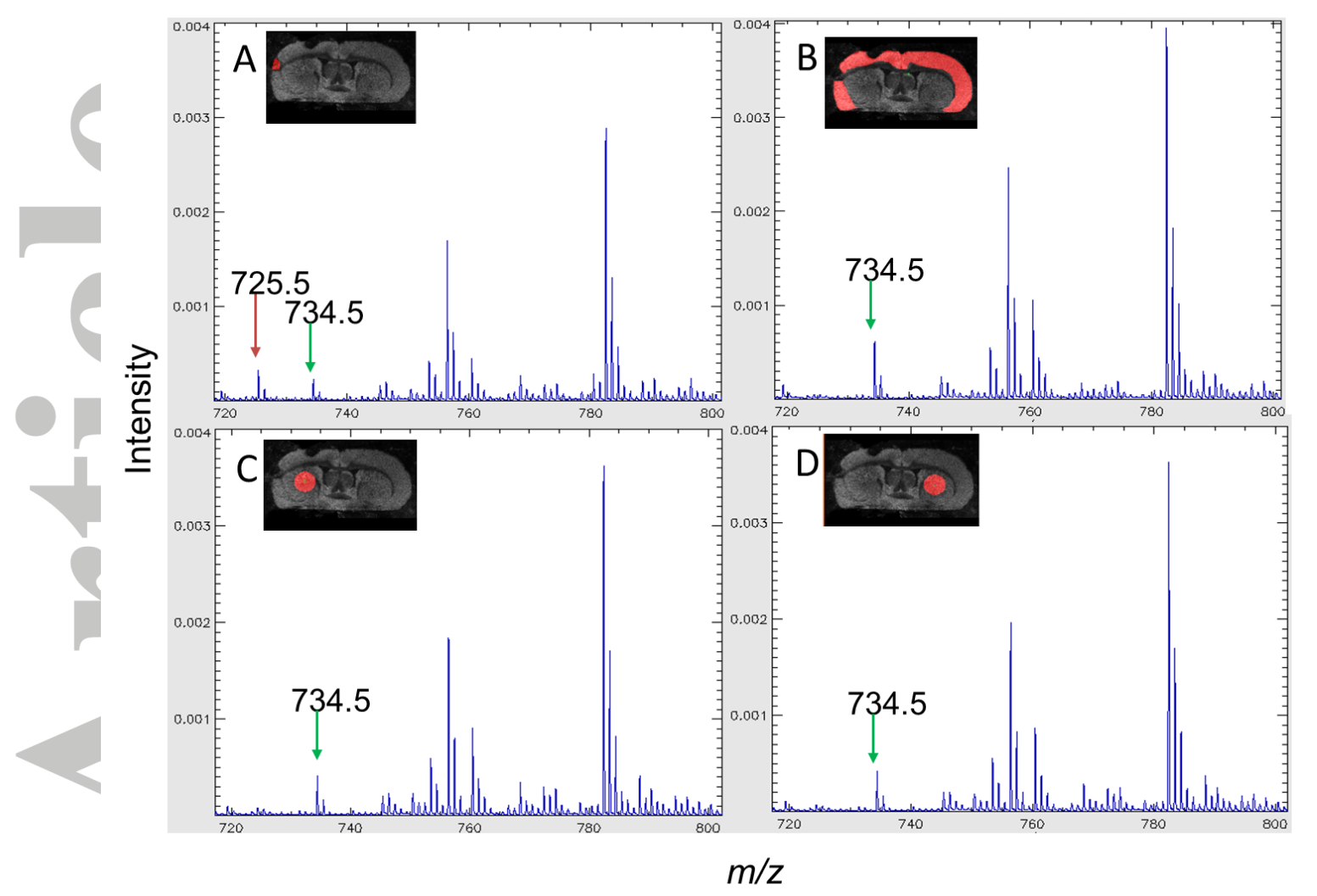

Fig. 3. MALDI-MS spectra of lipids in the scar region (A), isocortex (B), cerebral nuclei in each hemisphere (C) and (D); corresponding ROIs are shown. Arrows indicate examples of peaks which differ between the scar region and healthy brain. 


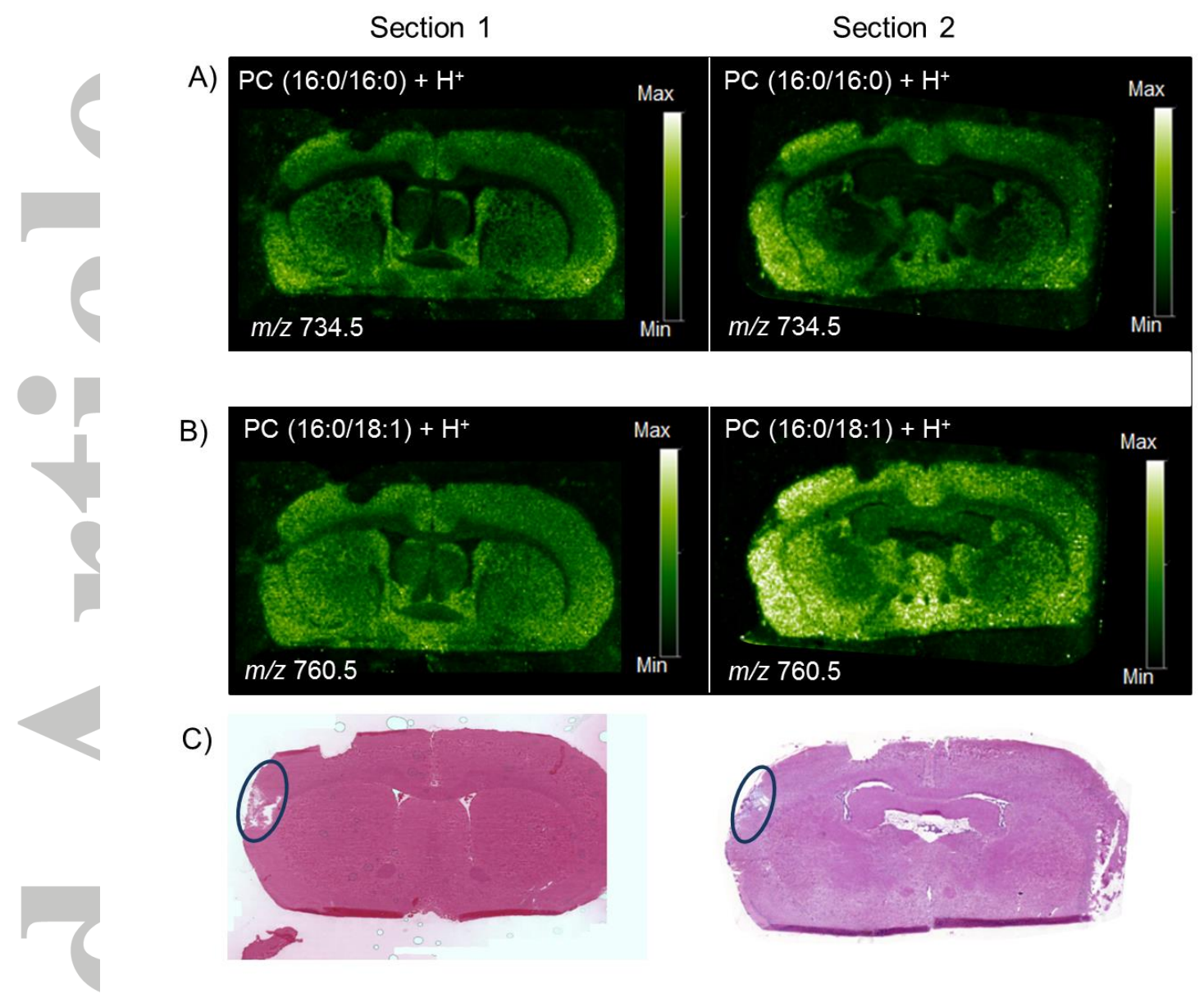

Fig. 4. MALDI-MS images of PC (16:0/16:0) (A) and PC (16:0/18:1) (B) in a coronal rat brain section three months post-stroke. Two different sections, (at 2 coronal brain levels) are shown, with corresponding H\&E stains (C). The scar region has been circled. 
Section 1

Section 2

A) $\mathrm{SM}(\mathrm{d} 18: 1 / 16: 0)+\mathrm{Na}^{+}$

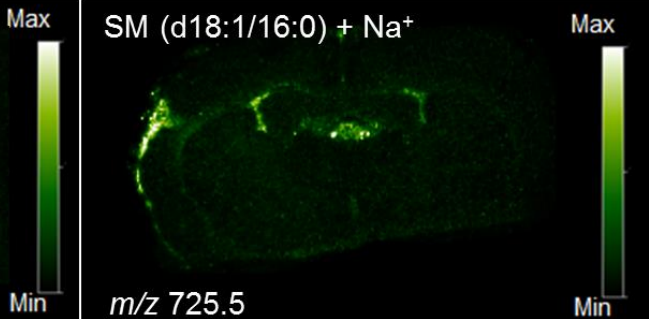

$m / z 725.5$

Min

$m / Z 725.5$

$\operatorname{Min}$

B)

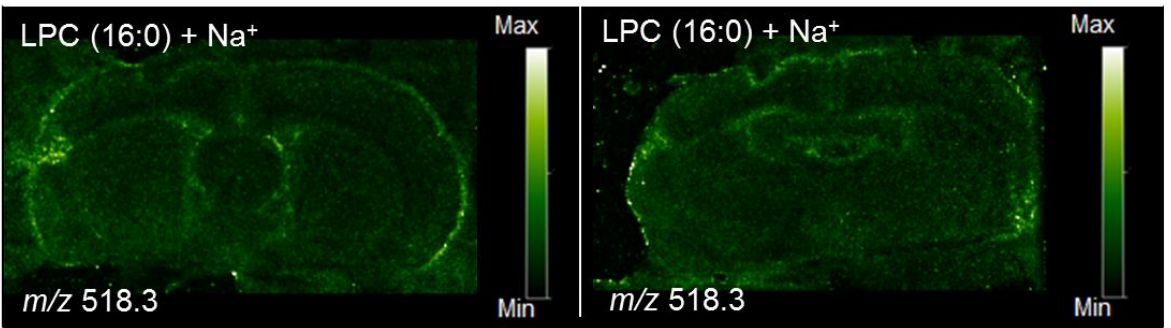

C)
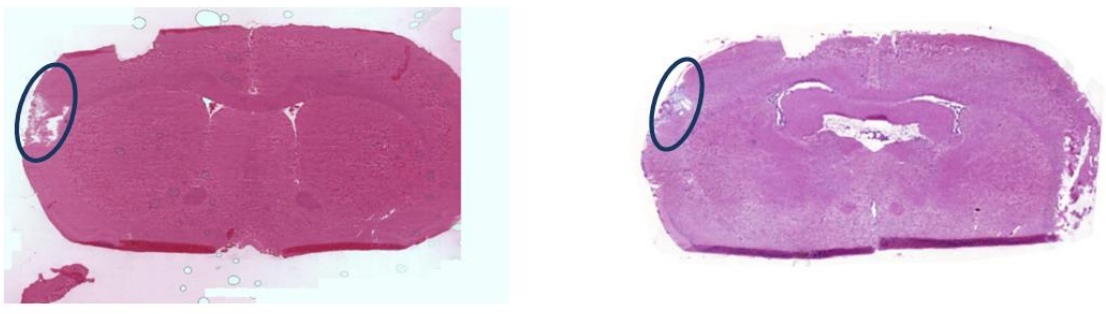

Fig. 5. MALDI-MS images of SM (d18:1/16:0) (A) and LPC (16:0) (B) in a coronal rat brain section three months post-stroke. Two different sections, (at 2 coronal brain levels) are shown, with corresponding H\&E stains (C). The scar region has been circled.

This article is protected by copyright. All rights reserved. 


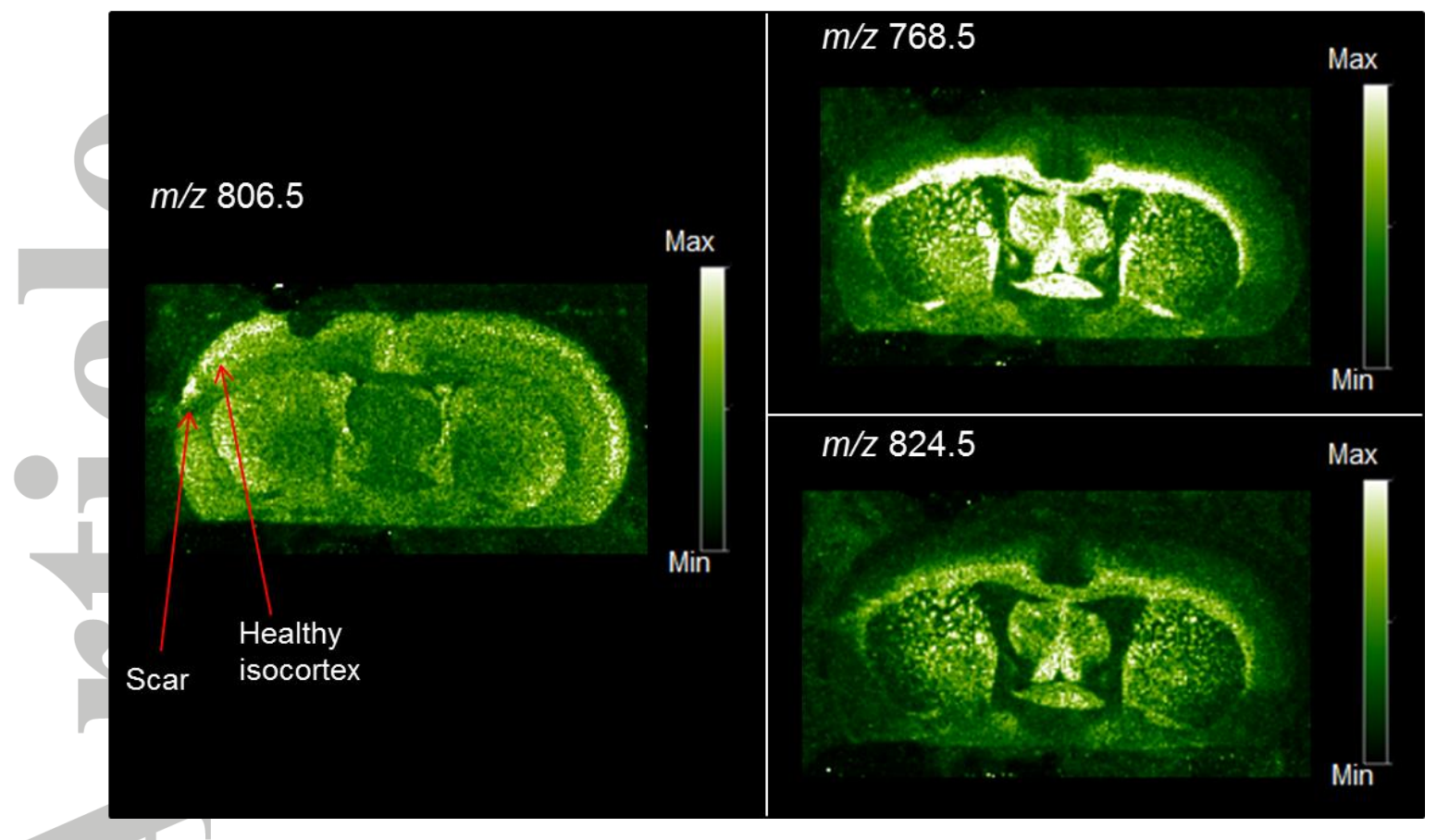

Fig. 6. MALDI-MS images of unidentified peaks localising in the scar region of a coronal rat brain section three months post-stroke.

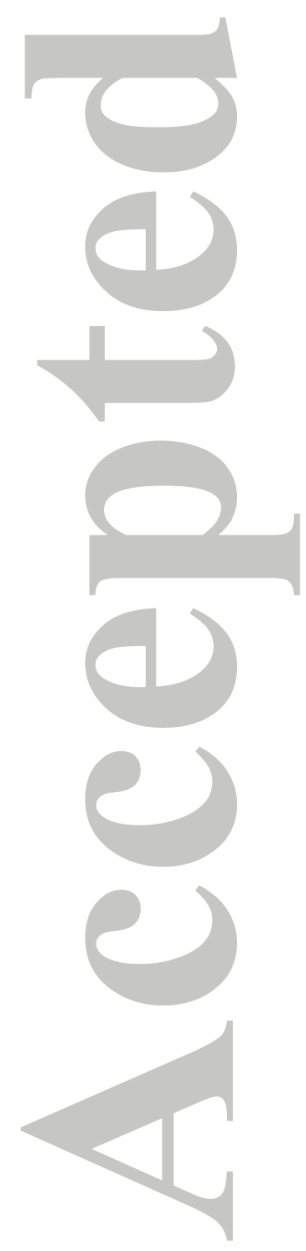

